University of South Carolina

Scholar Commons

8-7-2003

\title{
High-Field Measurements of Electron Decoherence Time in Metallic Nanowires: Switching off Magnetic Impurity Spins
}

\author{
P. Mohanty \\ Richard A. Webb \\ University of South Carolina - Columbia, webbra@mailbox.sc.edu
}

Follow this and additional works at: https://scholarcommons.sc.edu/phys_facpub

Part of the Physics Commons

Publication Info

Published in Physical Review Letters, Volume 91, Issue 6, 2003, pages 066604-1-066604-4.

Mohanty, P. and Webb, R.A. (2003). High-Field Measurements of Electron Decoherence Time in Metallic Nanowires: Switching off Magnetic Impurity Spins. Physical Review Letters, 91(6), 066604-1 - 066604-4. doi: 10.1103/PhysRevLett.91.066604 (c) 2003 The American Physical Society.

This Article is brought to you by the Physics and Astronomy, Department of at Scholar Commons. It has been accepted for inclusion in Faculty Publications by an authorized administrator of Scholar Commons. For more information, please contact digres@mailbox.sc.edu. 


\title{
High-Field Measurements of Electron Decoherence Time in Metallic Nanowires: Switching off Magnetic Impurity Spins
}

\author{
P. Mohanty ${ }^{1}$ and R. A. Webb ${ }^{2}$ \\ ${ }^{1}$ Department of Physics, Boston University, 590 Commonwealth Avenue, Boston, Massachusetts 02215, USA \\ ${ }^{2}$ Center for Superconductivity Research, University of Maryland, College Park, Maryland 20742, USA
}

(Received 12 February 2003; published 7 August 2003)

\begin{abstract}
We report low-temperature measurements of electron decoherence time in a series of pure gold wires, $18 \mathrm{~nm}$ thick and $30 \mathrm{~nm}$ wide. At fields up to $15 \mathrm{~T}$, large enough to polarize any concentration of magnetic impurity spins, conductance fluctuation measurements show almost no temperature dependence of the decoherence time below $300 \mathrm{mK}$, both in the correlation field for interference and the root-mean-square value of the fluctuations. Combined with previous low-field weak localization measurements on samples from similar material, our experiment suggests that the ubiquitous saturation of decoherence time in these samples is not due to any mechanism based on magnetic impurity spins.
\end{abstract}

DOI: 10.1103/PhysRevLett.91.066604

An electron has two distinct degrees of freedom, charge and spin. These two degrees of freedom define the electron wave function, its coherence as well as its decoherence. The charge degree of freedom contributes to decoherence due to its coupling to a randomly fluctuating electric field - or rather, a fluctuating vector potentialeither intrinsic or extrinsic. Likewise, the spin degree of freedom contributes to decoherence by its coupling to a randomly fluctuating magnetic field along the interfering electron path. Inside a nominally pure, metallic conductor, fluctuating magnetic fields could arise due to localized spins from the presumably unavoidable magnetic impurities. Because the interaction between the electron spin and the magnetic impurity spin is very complex, the problem of electron decoherence in the absence of magnetic impurities is fundamentally important [1-3].

Given the conceptual and formal difficulties with the understanding of the intrinsic mechanism of electronelectron interaction [4-6], it is natural to search for other extrinsic mechanisms that might cause temperature independent decoherence at low temperatures. The primary reason is that even with a single source of decoherence, it is possible to have many different mechanisms, which require different types of measurements to rule them out. Elsewhere, we have provided extensive checks for the effects of electron heating, high-frequency noise, twolevel defects, and magnetic impurities, and we have found that the observed saturation in our samples is not due to any of these mechanisms [2]. Here we report the results of a different type of measurement performed at high fields, which rules out an entire class of mechanisms based on magnetic impurity spins.

Magnetic impurity spins in dilute concentration give rise to the well-known Kondo effect [7] due to the screening of the impurity spin by the electron cloud below a characteristic Kondo temperature $T_{K}$ [8]. The process of spin flip in both the electron spin and the impurity spin is suppressed below $T_{K}$ in agreement with the Fermi liquid
PACS numbers: 72.15.-v, 71.30.+h, 73.20.Fz

theory of the Kondo effect [9]. As depicted in Fig. 1, electron decoherence time $\tau_{\phi}$ is also expected to diverge as $1 / T^{2}$ in regimes I and II, if the dominant decoherence mechanism is magnetic scattering. At $T \ll T_{K}$ (regime I), however, it is possible to recover the intrinsic decoherence time. For example, if the conventional theory of electron interaction describes the decoherence mechanism [10], $\tau_{\phi}$ will diverge as $T^{-2 / 3}$ in quasi-1D wires. If there is indeed an intrinsic decoherence mechanism, then $\tau_{\phi}$ will saturate. Above $T_{K}$, impurity spin fluctuates freely, and the scattering rate is described by the SuhlNagaoka interpolation between the high temperature limit $\left(T \gg T_{K}\right)$ and the low-temperature unitary limit $\left(T \ll T_{K}\right)$ of the Fermi liquid theory. Important to the work reported here is the observation that, above $T_{K}$ (regime III), the magnetic scattering time, and hence, $\tau_{\phi}$ show an extremely weak temperature dependence, mimicking saturation. Although a complete understanding of this regime is missing, this weak temperature dependence has been observed in a number of experiments [11]. At higher temperatures (regime IV), $\tau_{\phi}$ in systems with a known amount of magnetic impurities displays a faster dependence due to electron-phonon interaction.

It could be conjectured that the experimentally observed saturation of decoherence time in purely metallic samples could arise from the presence of an undetectable amount of magnetic impurities (from resistivity measurements, typically below $1 \mathrm{ppm}$ ) [12-14]. For instance, the observed saturation in pure samples could be due to the fact that $T_{K}$ lies below the lowest temperature of measurement $\left(T_{K}<T_{\text {lowest }}\right)$, and the experimental range of temperatures allows measurements only down to regime III. In addition, other nonequilibrium effects above $T_{K}$ could result in an apparent saturation as well as in a strong bias dependence [12-14]; because our measurements are done in the equilibrium regime with no observed bias dependence, the nonequilibrium mechanisms are not applicable to our experiments. 
$\tau_{\phi}$ (with magnetic spin)
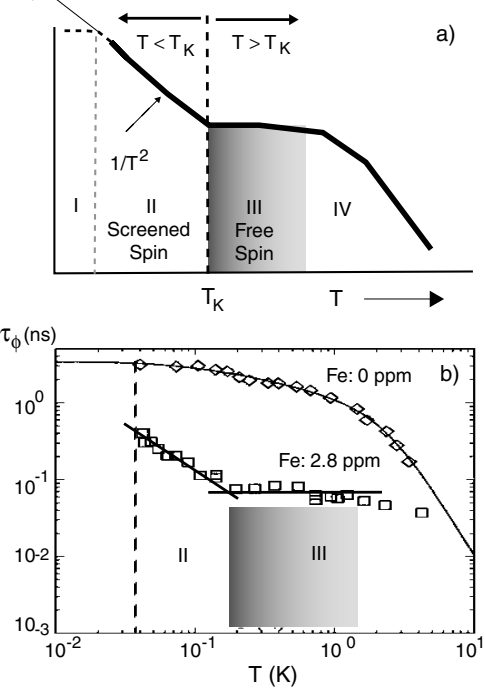

FIG. 1. Schematic dependence of electron decoherence time $\tau_{\phi}$ in a quasi-one-dimensional metallic wire in the presence of magnetic impurities. (a) The four regimes (depicted as I through IV) show distinct temperature dependence. At high temperatures (regime IV), phonon scattering dominates, giving rise to $\tau_{\phi} \sim T^{-3}$. Above $T_{K}$ (regime III), an apparent saturation is observed. Below $T_{K}$ (regime II), the spin is screened, and $\tau_{\phi}$ is dominated by the spin-flip time, $\tau_{\text {spin-flip }} \sim T^{-2}$. In regime I, $\tau_{\phi}$ could still be dominated by $\tau_{\text {spin-flip }}$, and diverge at $T=0$. (b) The top data set shows the saturation in a pure metallic quasi-1D wire (from Ref. [1]). The bottom data set is taken on the same sample after ion implantation of $2.8 \mathrm{ppm}$ of Fe impurities; it displays the expected dependence from regime II through IV.

Turning to experiments, recent measurements on nominally pure $\mathrm{Au}$ and $\mathrm{Cu}$ wires have revealed the presence of magnetic impurities in small concentrations. In a recent experiment from the Grenoble group [15], temperature dependence of $\tau_{\phi}$ displays the overall shape similar to the schematic diagram in Fig. 1(a), indicating the presence of magnetic impurities. However, at $T<T_{K}$ (regime II), data on many samples showed saturation down to $10 \mathrm{mK}$. In the same range, the resistivity showed a peak around $30 \mathrm{mK}(40 \mathrm{mK})$, indicative of a spin-glass system with an estimated $\mathrm{Fe}$ concentration of $15 \mathrm{ppm}$ (60 ppm) determined from the slope. The level of impurity concentration is an order of magnitude higher than the earlier experiments on pure samples [1] on the basis of the resistivity correction $(<1 \mathrm{ppm})$ and on samples [16] with a controlled amount of ion-implanted magnetic impurities $(\sim 3 \mathrm{ppm})$.

It is well known that magnetic impurities could be frozen by applying a large magnetic field to polarize the spins, both in the dilute Kondo regime, as reported in 1987 by Benoit et al. [17,18], and in the spin-glass regime [19]. In a recent experiment [20], measurements of Aharonov-Bohm oscillations at two different field ranges confirmed that the $\mathrm{Cu}$ samples under study could have an estimated amount of $\leq 1 \mathrm{ppm}$ of magnetic impurities. In this experiment, the Aharonov-Bohm oscillation amplitude could be enhanced by freezing the impurity spins at high magnetic fields, similar to the Benoit experiment on conductance fluctuations. A strong field dependence, as seen in the $\mathrm{Cu}$ samples, is indicative of magnetic impurities in small concentration but significant enough to dominate the temperature dependence of $\tau_{\phi}$. However, there are other experiments $[18,21,22]$ in which such a field dependence is not seen. This emphasizes the need to understand material choice and techniques of sample fabrication without contamination, or to devise new methods of probing intrinsic decoherence mechanisms independent of magnetic impurity spins.

The amplitude of Aharonov-Bohm (AB) oscillations in gold rings first observed in 1985 did not display such a strong field dependence up to 20 flux quanta (Fig. 1 of Ref. [21]). Subsequent measurements on similar samples did not show the supposed field dependence up to $8 \mathrm{~T}$ (see Fig. 1 of Ref. [22] as well as many other experiments discussed in Ref. [18]). Interestingly, Fig. 3 of Ref. [22] shows that the $T$ dependence of AB oscillations is primarily governed by $L_{T}$, and only weakly dependent on $L_{\phi}$. Strangely, the temperature dependence of $\tau_{\phi}$ from $\mathrm{AB}$ oscillations at high fields in the recent experiments on $\mathrm{Cu}$ rings [20] was not reported, which would have shed light on the real intrinsic mechanism independent of processes involving magnetic impurity spins. Finally, it is not clear as to why the peak-to-peak amplitude of oscillations even at the highest field [20] is more than an order of magnitude smaller than the usual value of $e^{2} / h$ (see, for instance, Fig. 10b of Ref. [18]).

In this Letter, we report measurements of electron decoherence time $\tau_{\phi}$ at high fields, obtained from conductance fluctuations. The samples are quasi-onedimensional gold wires with the following dimensions: $18 \mathrm{~nm}$ thick, $30 \mathrm{~nm}$ wide, and $20 \mu \mathrm{m}$ long, fabricated from gold with a purity of $99.9995 \%$. The three samples CF1, CF2, and CF3 have sample resistances of 2390, 2886 , and $3115 \Omega$, respectively. The diffusion constant in all these samples is approximately $0.005 \mathrm{~m}^{2} / \mathrm{s}$. Conductance fluctuations and weak localization are measured in these samples down to $39 \mathrm{mK}$ in fields up to $\pm 15 \mathrm{~T}$. The measurement currents used in these samples $(0.85,0.64$, and $0.99 \mathrm{nA}$, respectively) are not found to cause heating or any bias dependence down to the lowest measurement temperature of $39 \mathrm{mK}$.

Reproducible conductance fluctuations arise due to interference of electron paths inside the conductor [23]. For a completely phase-coherent sample, the fluctuation amplitude has the universal value $e^{2} / h$. However, finite temperature, characterized by the length $L_{T}=$ $\sqrt{\hbar D / k_{B} T}$, suppresses the fluctuations by energy averaging by a factor $\sqrt{E_{c} / k_{B} T}=L_{T} / L_{\phi}$, where $E_{c}=\hbar D / L_{\phi}^{2}$. Likewise, finite dephasing, characterized by $L_{\phi}=$ $\sqrt{D \tau_{\phi}}$, causes a further suppression by breaking the sample into statistically independent phase-coherent segments. In the limit of $L_{T} \leq L_{\phi} \leq L$, the rms value 

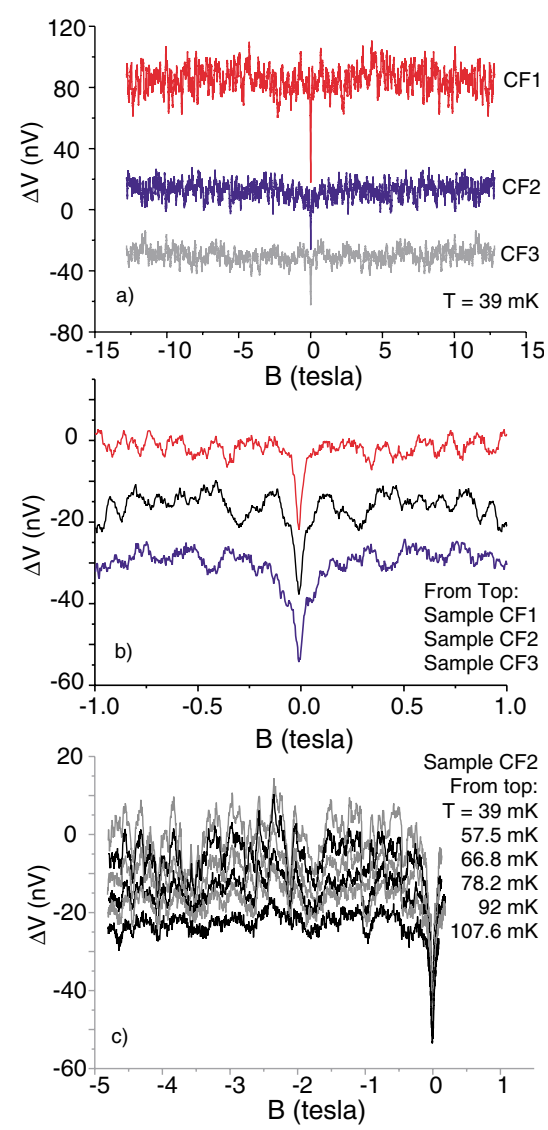

FIG. 2 (color online). (a) Magnetoresistance of three quasi1D gold wires (CF1, CF2, and CF3) over a field range of $\pm 15 \mathrm{~T}$, shown in the units of voltage. Weak antilocalization is observed at low fields because of strong spin-orbit scattering; fluctuations at higher fields are reproducible conductance fluctuations. (b) Magnetoresistance within a field span of $1 \mathrm{~T}$ is displayed. (c) Temperature dependence of magnetoresistance in a shorter field span is shown for sample CF2.

$[23,24]$ is $\delta G_{\mathrm{rms}} \simeq \sqrt{8 / 3 \pi} \frac{e^{2}}{h}\left(L_{\phi} / L\right)^{3 / 2}\left(L_{T} / L_{\phi}\right)$. Using this expression, $L_{\phi}$ and $\tau_{\phi}\left(=L_{\phi}^{2} / D\right)$ can be determined:

$$
L_{\phi} \simeq \frac{3 \pi}{8} \frac{L^{3}}{L_{T}^{2}}\left(\frac{\delta G_{\mathrm{rms}}}{e^{2} / h}\right)^{2} .
$$

$L_{\phi}$ can also be obtained from the autocorrelation function of the fluctuations. An important aspect of this method is that the autocorrelation function does not involve energy averaging or the $L_{T}$ dependence. The correlation field $B_{c}$ is the field lag $\Delta B$ where the normalized autocorrelation function is half of its zero-field value. Physically, $B_{c}$ is the field required to apply a single flux quantum $h / e$ through the phase-coherent area $w L_{\phi}[25]$, or

$$
L_{\phi} \simeq C \frac{h / e}{w B_{c}} \quad(C \simeq 0.95) .
$$

Figure 2(a) displays magnetoresistance traces for the three quasi-1D gold wires. The sample length of $20 \mu \mathrm{m}$ is designed to be roughly $5 L_{\phi}$ long at $39 \mathrm{mK}$. Figure 2(b)
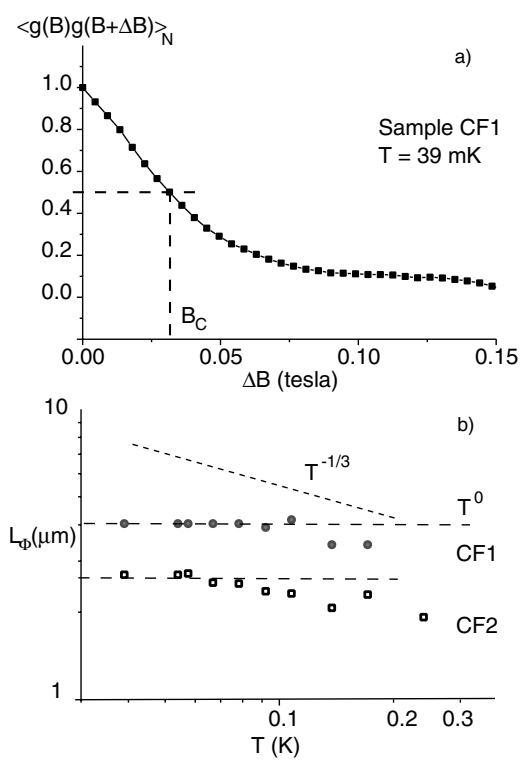

FIG. 3. (a) Normalized autocorrelation function calculated from the conductance fluctuations above a field magnitude of $1 \mathrm{~T}$. (b) Temperature dependence of $L_{\phi}$ for the samples CF1 and $\mathrm{CF} 2$, determined from the temperature dependence of the correlation field $B_{c}$, shows the saturation.

shows the low-field part, containing weak antilocalization contributions. For reference, $L_{\phi}$ determined from weak antilocalization measurement at $39 \mathrm{mK}$ for sample CF1 is $4 \mu \mathrm{m}$. Figure 2(c) shows the temperature dependence of conductance fluctuations in the sample CF2 at low temperatures. Apart from the weak antilocalization contributions, the amplitude of conductance fluctuations shown in Fig. 2 does not display any obvious change as a function of magnetic field that would be expected due to the polarization of the impurity spins [17]. Note that the polarization of magnetic impurities in the samples in Ref. [20] resulted in an order of magnitude change in the amplitude of Aharonov-Bohm oscillations. Figure 3 displays the autocorrelation of conductance fluctuations above $1 \mathrm{~T}$ for the sample CF1. Figure 3(b) shows temperature dependence of $L_{\phi}$ for CF1 and CF2 below $200 \mathrm{mK}$.

Mean conductance of the two samples CF1 and CF2 is plotted as a function of $T^{-1 / 2}$ in Fig. 4(a) along with the linear fits, expected from electron-electron interaction so electron temperature is well defined down to $39 \mathrm{mK}$. Figure 4(b) displays the temperature dependence of the rms value of conductance fluctuations for CF1 and CF2. The solid lines show the $T^{-1 / 2}$ dependence, expected from energy averaging $\left(L_{T}\right)$ of the fluctuations. $L_{\phi}(T)$ determined with Eq. (1) shows the saturation, consistent with Fig. 3(b). The weak dependence in $L_{\phi}$ or $\tau_{\phi}$ is obtained irrespective of the low-field cutoff of $1 \mathrm{~T}$.

A number of important observations emerge from our measurements: (i) in pure metallic quasi-1D gold nanowires, $L_{\phi}$ or $\tau_{\phi}$ at high fields saturates at low temperatures; (ii) because magnetic impurity spins at any level of 

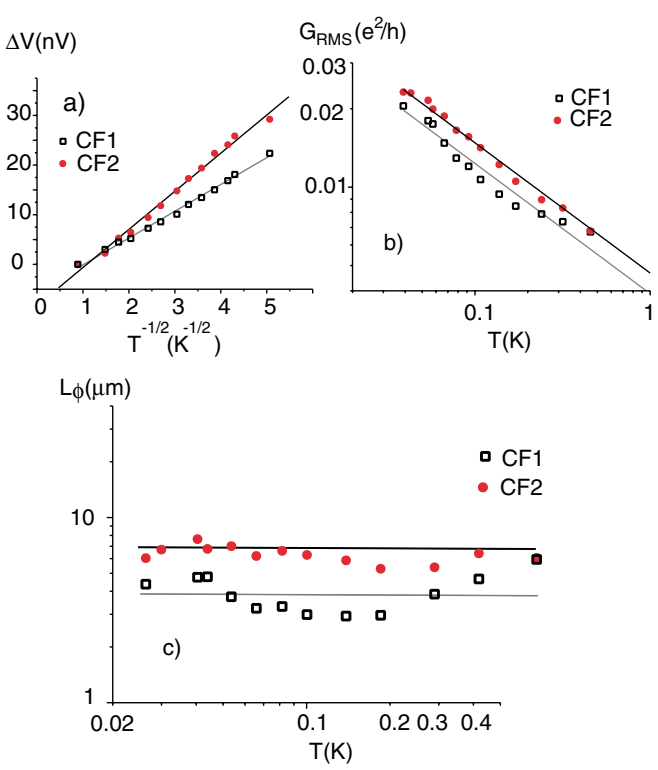

FIG. 4 (color online). (a) Temperature dependence of the mean conductance of the two samples CF1 and CF2 (in units of volts) is plotted as a function of $T^{-1 / 2}$. The lines are linear fits to $T^{-1 / 2}$, expected from the electron interaction correction. (b) The rms values of the fluctuations above the field magnitude of $1 \mathrm{~T}$ are shown. The lines are functional forms for $T^{-1 / 2}$. (c) Temperature dependence of phase decoherence length $L_{\phi}$ determined from the rms values of the fluctuations shown in (b).

concentration would be frozen at high fields, the dominant mechanism of decoherence in our quasi-1D gold nanowires does not involve magnetic impurities; (iii) using our fabrication techniques and material $(A u)$, not-toodisordered, purely metallic, quasi-1D gold nanowires always show a saturation in $L_{\phi}$ or $\tau_{\phi}$ at high fields and low temperatures.

In conclusion, the temperature dependence of our conductance fluctuation measurements over the very large magnetic field range of $\pm 15 \mathrm{~T}$ demonstrate that the decoherence time determined from the correlation field and the rms value of the fluctuations is temperature independent below $300 \mathrm{mK}$. These measurements reveal that there must be an additional source of intrinsic decoherence since it is believed that magnetic impurities at very low temperatures and very high fields cannot cause decoherence because of their polarization along the field.

For support of this work, P. M. thanks Boston University and Sloan Foundation, and R. A.W. acknowledges NSF (DMR 0103223), NSA, and ARDA (MDA90401C0903).

[1] P. Mohanty, E. M. Q. Jariwala, and R. A. Webb, Phys. Rev. Lett. 78, 3366 (1997).

[2] P. Mohanty, in Complexity from Microscopic to Macroscopic Scales: Coherence and Large Deviations, edited by A.T. Skjeltorp and T. Vicsek (Kluwer, Dordrecht, 2002); cond-mat/0205274.

[3] J. J. Lin and J. Bird, J. Phys. Condens. Matter 14, R501R596 (2002).

[4] D. S. Golubev and A. D. Zaikin, Phys. Rev. Lett. 81, 1074 (1998); Phys. Rev. B 59, 9195 (1999); D. Golubev, A. D. Zaikin, and G. Schoen, J. Low Temp. Phys. 126, 1355 (2002).

[5] I. L. Aleiner, B. L. Altshuler, and M. Gershenson, Waves Random Media 9, 201 (1999).

[6] J. von Delft, cond-mat/0210644; F. Marquardt, condmat/0207692.

[7] A. C. Hewson, The Kondo Problem to Heavy Fermions (Cambridge University Press, Cambridge, 1993).

[8] (a) The single-impurity Kondo effect can be complicated by the coupling between the impurity spins via the RKKY interaction, governed by the interimpurity distance or the impurity concentration. A spin glass is formed below a characteristic glass temperature $T_{g}$. For concentrations under $5 \mathrm{ppm}, T_{g}$ in a AuFe system is estimated to lie much below $40 \mathrm{mK}$. Therefore, the complications due to spin-glass formation can be safely ignored in the present experiment. (b) Higher-order effects such as two-channel Kondo effect or multichannel Kondo effect are not relevant to the present experiment.

[9] P. Nozieres, J. Low Temp. Phys. 17, 31 (1974).

[10] B. L. Altshuler, A. G. Aronov, and D. E. Khmelnitskii, J. Phys. C 15, 7367 (1982).

[11] R. P. Peters, G. Bergmann, and R. M. Mueller, Phys. Rev. Lett. 58, 1964 (1987); C. V. Haesendonck, J. Vranken, and Y. Bruynseraede, Phys. Rev. Lett. 58, 1968 (1987).

[12] A. Kaminski and L. Glazman, Phys. Rev. Lett. 86, 2400 (2001).

[13] J. S. Meyer, V. I. Falko, and B. S. Altshuler, cond-mat/ 0206024.

[14] G. Goeppert et al., Phys. Rev. B 66, 195328 (2002); G. Goppert and H. Grabert, Phys. Rev. B 64, 33301 (2001); J. Kroha, cond-mat/0102185; J. Kroha and A Zawadowski, Phys. Rev. Lett. 88, 176803 (2002); A. Anthore et al., Phys. Rev. Lett. 90, 076806 (2003).

[15] F. Shopfer et al., Phys. Rev. Lett. 90, 056801 (2003).

[16] P. Mohanty and R. A. Webb, Phys. Rev. Lett. 84, 4481 (2000).

[17] A. Benoit et al., in Anderson Localization, edited by T. Ando and H. Fukuyama (Springer-Verlag, Berlin, 1988); also discussed in Ref. [18].

[18] S. Washburn and R. A. Webb, Rep. Prog. Phys. 55, 1311 (1992).

[19] L. Levy (private communication).

[20] F. Pierre and N. O. Birge, Phys. Rev. Lett. 89, 206804 (2002); F. Pierre et al., cond-mat/0302235.

[21] R. A. Webb et al., Phys. Rev. Lett. 54, 2696 (1985).

[22] S. Washburn et al., Phys. Rev. B 32, R4789 (1985).

[23] P. A. Lee, A. D. Stone, and H. Fukuyama, Phys. Rev. B 35, 1039 (1987).

[24] V. Chandrasekhar, P. Santhanam, and D. E. Prober, Phys. Rev. B 42, 6823 (1990); we ignore the spin-orbit triplet term as $\mathrm{Au}$ has strong spin-orbit scattering, $L_{\text {so }} \ll L_{\phi}$.

[25] C.W. J. Beenakker and H. van Houten, Phys. Rev. B 37, 6544 (1988). 